\title{
Electromechanical Coupling in Cardiomyocytes Depends on Its Electrotonic Interaction With Fibroblasts: Simulation Study
}

\author{
Anastasia Bazhutina ${ }^{1}$, Nathalie Balakina-Vikulova ${ }^{1,2}$, Leonid Katsnelson ${ }^{1,2}$, Alexander V Panfilov ${ }^{1,3}$, \\ Olga Solovyova ${ }^{1,2}$ \\ ${ }^{1}$ Ural Federal University, Ekaterinburg, Russia \\ ${ }^{2}$ Institute of Immunology and Physiology, Ekaterinburg, Russia \\ ${ }^{3}$ Ghent University, Ghent, Belgium
}

\begin{abstract}
Cardiac fibroblasts are interspersed within mammalian cardiac tissue. Being mechanically passive they have own changeable membrane potential. They communicate electrically with cardiomyocytes via gap junctions and thus may affect electrical and mechanical activity of the latter. This influence may be analyzed in mathematical models. Mathematical models of fibroblast-cardiomyocyte interaction developed earlier allow anyone to study only electrical responses of the cardiomyocytes and fibroblasts to their electrical interaction. In this work, we investigated the fibroblast influence on the cardiomyocyte mechanics during afterloaded contractions. In numerical experiments, we observed significant changes in cardiomyocyte action potential duration, isometric force and load-dependent relaxation with an increase in the number of fibroblasts connected with the cardiomyocyte.
\end{abstract}

\section{Introduction}

In mammalian heart tissue, fibroblasts along with cardiomyocytes are one of the main types of cardiac cells. Cardiac fibroblasts are about 5-10 times smaller than cardiomyocytes, but their number in the ventricle is about two times higher than the cardiomyocytes [1], but only cardiomyocytes provide contraction of the heart in response to electrical stimulation of the heart tissue. Cardiac fibroblasts account for $10 \%$ of the volume of all cardiac cells due to its small size. In the membrane of fibroblasts there are ion channels that provide the resting potential in these cells. Cardiomyocytes and fibroblasts are in contact through high-resistance gap junctions. The current through the gap junctions provides mutual electrotonic influence on the membrane potential of the cells of both types [2].

Fibroblasts are cells of the connective tissue and affect myocardial remodeling. However, the mechanism of their interaction with the cardiomyocyte and the effect on its electromechanical activity has not been fully studied. Earlier, several mathematical models were developed for the interaction of fibroblasts with cardiomyocytes, one of which is the MacCannell2007 model [3]. In that model, the electrical interaction of the fibroblast and cardiomyocyte was described by a system of differential equations. There, the effect of the number of attached fibroblasts to the cardiomyocyte on the electrical function of cardiomyocyte was studied. But the effect of the combination of fibroblasts with cardiomyocyte on the mechanics of cardiomyocyte was not considered in that model. In our work we study this influence. In addition, in our model, we studied the reverse effect of the mechanics of cardiomyocyte on the electrical interaction of cardiomyocyte with fibroblasts.

\section{Methods}

\subsection{Model of fibroblast-cardiomyocyte interaction}

For the modeling of the fibroblast-cardiomyocyte electrical interaction we used the MacCannell2007 (MC07) [3]. The differential equation for the transmembrane potential in the $i$-th coupled fibroblast is:

$$
\frac{d V_{f i}}{d t}=-\frac{1}{C_{m f}}\left[I_{f i}\left(V_{f i}, t\right)+G_{g a p}\left(V_{f i}-V_{m}\right)\right]
$$

where $V_{f i}$ is membrane potential on the fibroblast membrane, $C_{m f}$ is the fibroblast membrane capacitance $(6.3 p F), \quad I_{f i}$ is a sum of transmembrane currents across the fibroblast membrane, $G_{g a p}$ is the fibroblastcardiomyocyte conductance ( $3 n S), V_{m}$ is an action potential on the cardiomyocyte membrane. Four membrane ionic currents for fibroblasts are considered in the MC07 model: a time and voltage-dependent "delayed-rectifier" $K^{+}$current $I_{K_{v}}$, an inward-rectifying $K^{+}$current $I_{K 1}$, a $\mathrm{Na}^{+}-\mathrm{K}^{+}$pump current $\mathrm{I}_{\mathrm{NaK}}$, a background $\mathrm{Na}^{+}$"leak" current $I_{b, N a}$. The differential equation for the transmembrane potential in the cardiomyocyte is: 

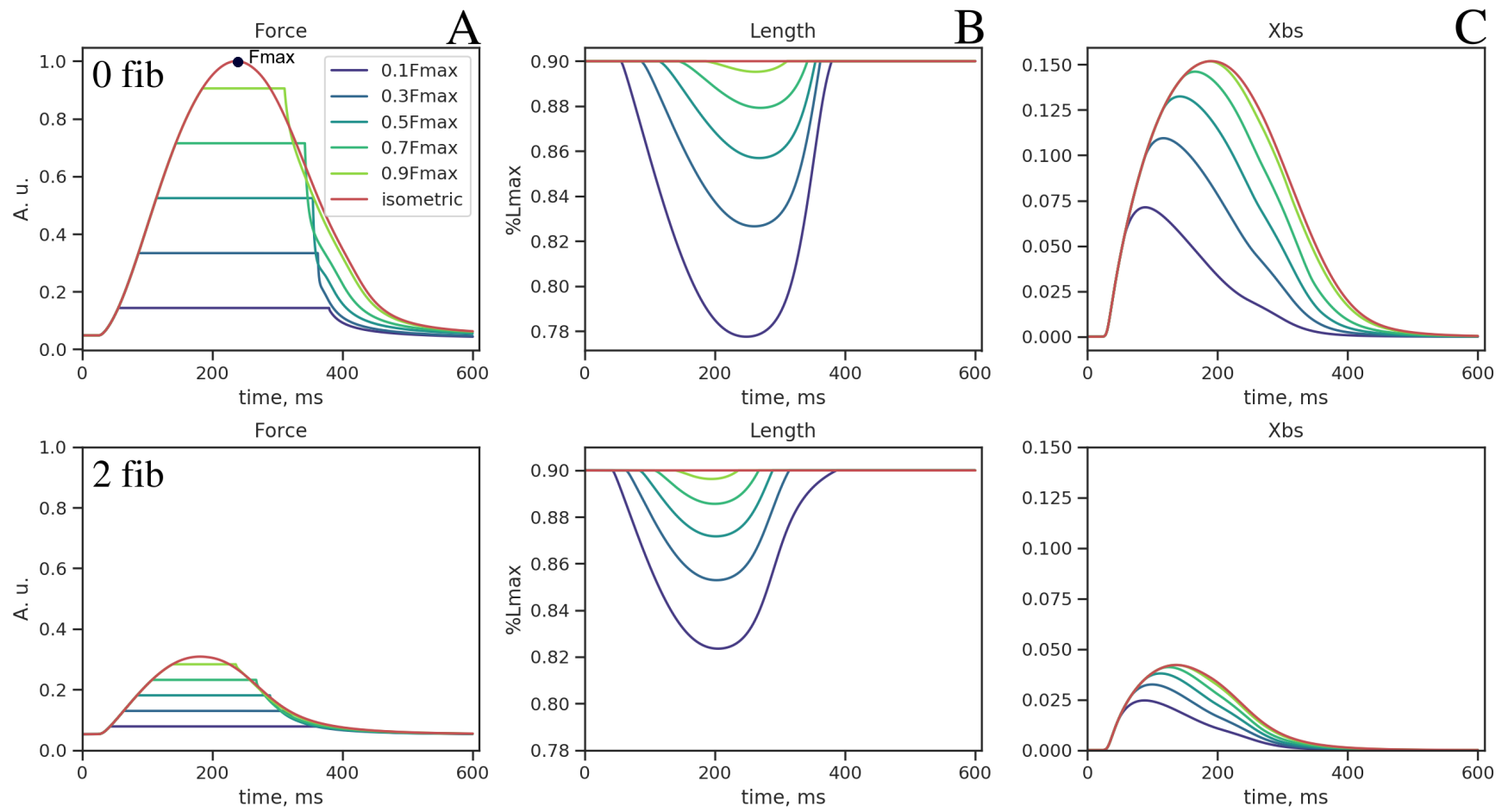

Figure 1. Mechanical activity of the virtual cardiomyocyte during afterloaded twitches in the absence of coupled fibroblasts ( $n=0$, top panels) and with two coupled fibroblasts $(n=2$, bottom panels). A. Force generated by the virtual cardiomyocyte. Force values are normalized to the peak value of the isometric force when $n=0$. B. Deformation of the virtual cardiomyocyte relatively to $\operatorname{Lmax}$. C. Fraction of force generating crossbridges $(X b s)$.
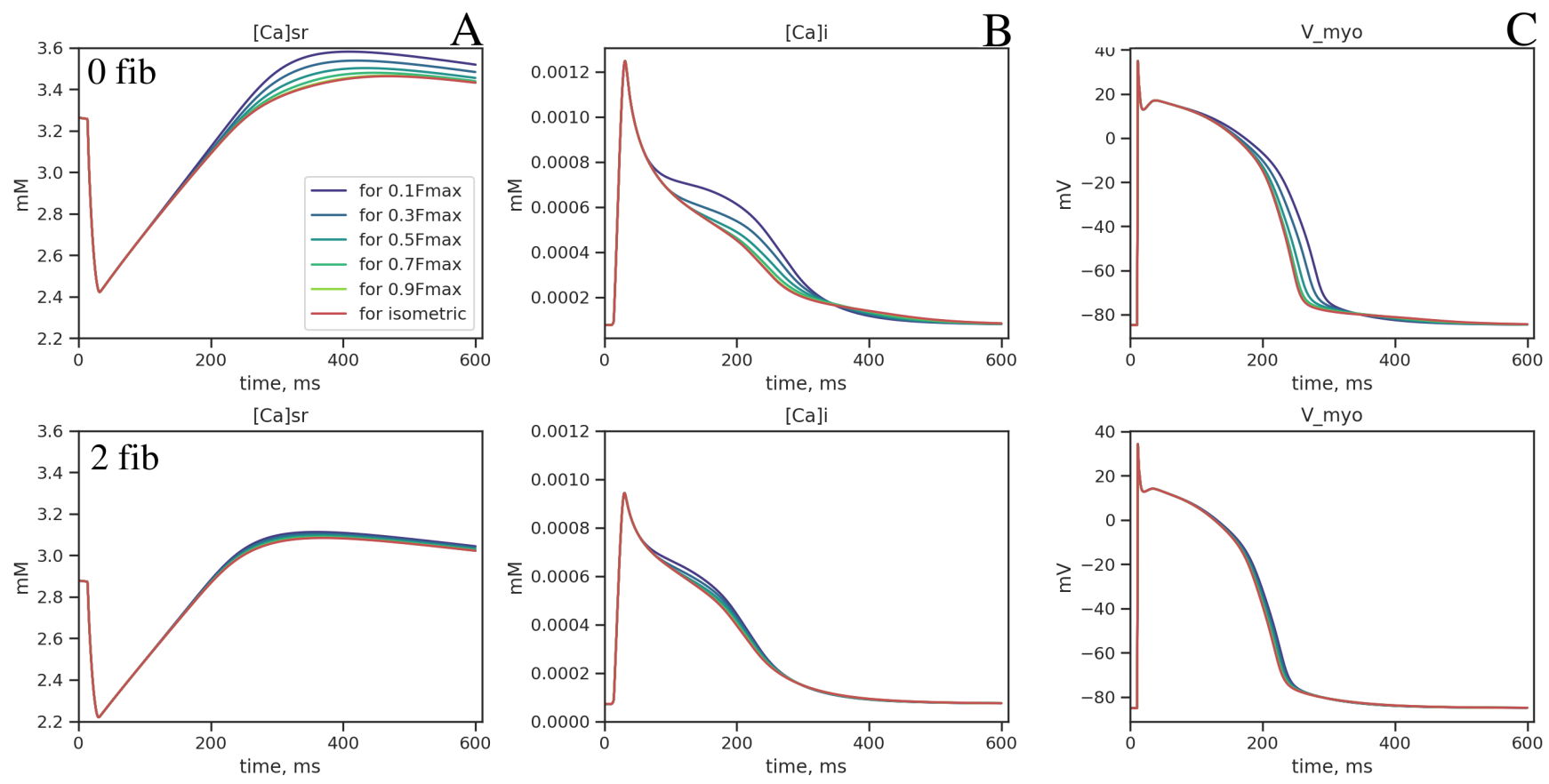

Figure 2. Afterload-dependent changes in calcium concentration in sarcoplasmic reticulum (A), in cytosole (B) and changes in membrane potential (C) during afterloaded twitches in the absence of coupled fibroblasts $(n=0$, top panels) and with two coupled fibroblasts ( $n=2$, bottom panels). 


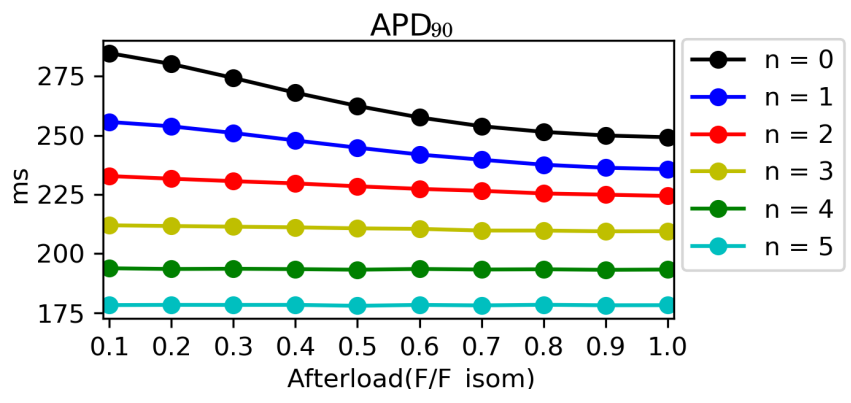

Figure 3. Effect of loads on action potential duration $\left(A P D_{90}\right)$ of the virtual cardiomyocyte during afterloaded twitches in the absence of coupled fibroblasts $(n=0)$ and with coupled fibroblasts ( $n$ varied from 1 to 5). Afterload values for each $n$ are given in relation to the respective peak value of the isometric force.

$$
\frac{d V_{m}}{d t}=-\frac{1}{C_{m m}}\left[I_{m}\left(V_{m}, t\right)+\sum_{i=1}^{n} G_{g a p}\left(V_{m}-V_{f i}\right)\right]
$$

where $C_{m m}$ is the myocyte membrane capacitance $(185 \mathrm{pF}), I_{m}$ is sum of cardiomyocyte transmembrane currents from the ten Tusscher-Panfilov (TP) model [4] of the human ventricular action potential, $i \in\{1, \ldots, n\}, n$ is the total number of fibroblasts connected with the cardiomyocyte.

\subsection{The fibroblast-cardiomyocyte model with the myocyte mechanics included}

We have earlier developed the TP+M model [5], which combines TP model with the module of the cardiomyocyte mechanical activity from the Ekaterinburg-Oxford electromechanical model [6]. The TP+M model reproduces electromechanical coupling and mechano-electric feedbacks in the cardiomyocytes.

We substitute the original TP model with the TP+M one in the MC07 model to consider electrotonic interaction effects on cardiomyocyte electromechanics. Contraction of simulated cardiomyocytes was invoked with pacing rate of $1 \mathrm{~Hz}$. The number of identical fibroblasts coupled to the single cardiomyocyte was varied in numerical experiments from 1 to 5 . We simulated isometric and isotonic modes of contraction. The shortening phase of isotonic mode is an in vitro experimental analogue of blood extrusion during the heart contraction.

For the beginning we simulated steady-state isometric twitches at the length of $0.9 L_{\max }$ (where $L_{\max }$ is the length at which the maximum isometric force is generated). Then isotonic twitches under various afterloads were performed, each starting from the isometric steadystate initial conditions.
The TP+M model includes descriptions of intracellular mechanisms underlying both mechano-calcium and mechano-electric feedbacks in cardiomyocytes, which allows myocardium to tune its contractile and electrical activity to the mechanical conditions of the contractions. These feedbacks manifest themselves, in particular, in such experimental phenomena as myocardium load-dependent relaxation. Briefly, the effect of load dependence is the speeding up of isotonic twitches (especially their relaxation phases) under medium and small afterloads as compared to the isometric one, as shown in Fig. 1. In these isotonic modes of contractions, the afterload value also affects the shape and duration of both calcium transients and action potentials in the cardiomyocyte. All these effects result from significant shortening of sarcomeres during isotonic contractions. The shortening intensifies decay of the calcium-troponin complexes, i.e. inactivates the cardiomyocyte.

\section{Results}

We have shown that the action potential duration in isometric contraction (Fig. 2C; $A P D_{90}$, Fig. 3) in the cardiomyocyte and respective duration of the repolarization phase in the fibroblast membrane potential decreased with an increase in connected fibroblast numbers [7]. These results are consistent with that of the MC07 simulations [3].

Despite the absence of the cardiomyocyte-fibroblast mechanical interaction in the model, we observed changes in the contractile function of the cardiomyocyte electrically coupled with the fibroblasts. Significant decrease in the cardiomyocyte isometric force peak was observed with an increase in the number of the fibroblasts (Fig. 1A). Even the coupling of 2 fibroblasts to the cardiomyocyte results in about $70 \%$ decrease in the peak force. The total contractile cycle duration shortened in the cardiomyocyte with an increase in the number of fibroblasts (Fig. 1). The attenuation of the myocyte mechanical activity (Fig. 1) was connected with the calcium unloading from the sarcoplasmic reticulum when the myocyte was coupled to fibroblasts (Fig. 2A). This, in turn, led to decreasing of the myocyte shortening magnitude in afterload contractions. Therefore, the influence of dynamic changes of the myocyte length on the calcium-troponin break-up also reduced. As a result of differences in the calcium transition duration, otherness in $A P D_{90}$ values became insignificant (Fig. 2, bottom row) during contractions under different afterloads, also the load-dependent mechanical effect completely disappeared (Fig. 1, bottom row) already with 2 or more connected fibroblasts.

So significant change in the myocardium mechanical performance due to the local environment seems to fits well with the overall pattern of the heart muscle responses to the specific conditions of its contractions. For exam- 
ple, it is well known that $10-20 \%$ decrease in the initial muscle lengthening results in the dramatic (up to tenfold) decrease in the peak isometric force [8].

The load-dependence of the isotonic twitches and of both action potential and $\mathrm{Ca}^{2+}$ transient durations almost disappeared in the TP+M model even for 2 coupled fibroblasts (Fig. 1 and Fig. 2) and vanished totally for the bigger number of the fibroblasts. With the increasing number of coupled fibroblasts the calcium concentration in the sarcoplasmic reticulum of the cardiomyocyte decreased in isometric steady-state (Fig. 2A). This, in turn, led to a decrease in $\mathrm{Ca}^{2+}$ transients (Fig. 2B), in $\mathrm{Ca}^{2+}$-troponin $\mathrm{C}$ concentration and in cross-bridge concentration (Fig 1C), and thus resulted in the loss of the contractility (Fig. 1B). The latter in particular means very small shortening with respect to the initial length during any isotonic twitch. In other words, length dependent inactivation of the twitch turned out quite insignificant. Thus, load dependence disappeared.

\subsection{Conclusions}

In this work, we presented results obtained in the mathematical model, where not only electrotonic interaction of a fibroblast and a cardiomyocyte is taken into account, but mechanical activity of the cardiomyocyte was incorporated in the model. As well as in the MC07 model, our numerical experiments displayed significant effect of the fibroblastcardiomyocytes electrical coupling on the electrical function of the cardiomyocyte, revealing a decrease in the cardiomyocyte action potential duration due to an increase in a number of the coupled fibroblasts.

We also considered mechanical response of the cardiomyocyte to this coupling. We showed that electrotonic interaction with fibroblasts depressed mechanical activity: the bigger the number of coupled fibroblasts, the lower the activity.

Obtained results suggest that an increase in the number of fibroblasts, in pathological conditions and during the aging may depress both electrical and mechanical heart function. Moreover, according to the predictions of our model, an increase in the number of fibroblasts suppresses the much-needed contour of the regulation of contractile activity of cardiomyocytes, namely intracellular mechanoelectric feedback, which allows a healthy heart to adapt to the changing mechanical conditions of contractions.

Noteworthy, that there is no possibility to determine exactly the actual number of fibroblasts coupled to a cardiomyocyte, either on average or by region due to the lack of the respective morphological data in situ. In such a case this being all the more important to model and analyze the influence of various number of fibroblasts coupled to a cardiomyocyte on the electrical and mechanical function of the latter. MC07 [3] performed such modeling and analy- sis for the electrical function of the cardiomyocyte. In our work, in addition to the electrical function, we studied the effect of the mechanical function of the cardiomyocyte.

\section{Acknowledgments}

The work was carried out within the framework of the IIF UrB RAS theme No AAAA-A18-118020590031-8, AAAA-A18-118020590134-6 and was supported by the Russian Foundation for Basic Research (18-29-13008, 1801-00059, 18-015-00368) and by RF Government Act \#211 of March 16, 2013 (agreement 02.A03.21.0006).

\section{References}

[1] Banerjee I, Fuseler JW, Price RL, Borg TK, Baudino TA. Determination of cell type and number during cardiac development in the neonatal and adult rat and mouse. American Journal of Physiology Heart and Circulatory Physiology 2007;293(3):H1883-H1891.

[2] Zhang P, Su J, Mende U. Cross talk between cardiac myocytes and fibroblasts: from multiscale investigative approaches to mechanisms and functional consequences. American Journal of Physiology Heart and Circulatory Physiology 2012;303(12):H1385-H1396.

[3] MacCannell KA, Bazzazi H, Chilton L, Shibukawa Y, Clark RB, Giles WR. A mathematical model of electrotonic interactions between ventricular myocytes and fibroblasts. Biophysical journal 2007;92(11):4121-4132.

[4] Ten Tusscher K, Noble D, Noble PJ, Panfilov AV. A model for human ventricular tissue. American Journal of Physiology Heart and Circulatory Physiology 2004; 286(4):H1573H1589.

[5] Balakina-Vikulova N, Solovyova O, Panfilov A, Katsnelson L. Mechano-electric feedbacks in a new model of the excitation-contraction coupling in human cardiomyocytes. Computing 2018;45:1.

[6] Sulman T, Katsnelson LB, Solovyova O, Markhasin VS. Mathematical modeling of mechanically modulated rhythm disturbances in homogeneous and heterogeneous myocardium with attenuated activity of na+-k+ pump. Bulletin of mathematical biology 2008;70(3):910-949.

[7] Bazhutina A, Balakina-Vikulova N, Katsnelson LB, Solovyova O. Mathematical model of electrotonic interaction between mechanically active cardiomyocyte and fibroblasts. USBERIT 2019;(in press).

[8] Allen D, Kentish J. The cellular basis of the length-tension relation in cardiac muscle. Journal of molecular and cellular cardiology 1985;17(9):821-840.

Address for correspondence:

Anastasia Bazhutina

Ural Federal University,

Mira 19, Ekaterinburg 620002, Russia

E-mail: banas49@mail.ru 